Pontifícia Universidade C $_{\text {atólica }}$

DO RIO DE JANEIRO

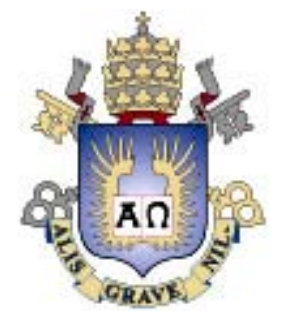

Welber Gianini Quirino

Produção e caracterização de dispositivos orgânicos eletroluminescentes (OLEDs) baseados em complexos $\beta$-dicetonatos de Terras-Raras

Tese apresentada como requisito parcial para obtenção do título de Doutor pelo Programa de PósGraduação em Física da PUC-Rio.

Orientador: Prof. Marco Cremona.

Rio de Janeiro, 16 de Março de 2007. 
Welber Gianini Quirino

\section{Produção e caracterização de dispositivos orgânicos eletroluminescentes (OLEDs) baseados em complexos $\beta$-dicetonatos de Terras-Raras}

Tese apresentada como requisito parcial para obtenção do título de Doutor pelo Programa de Pós-Graduação em Física da PUC-Rio. Aprovada pela Comissão Examinadora abaixo assinada.

Prof. Marco Cremona

Orientador

Departamento de Física - PUC-Rio

Profa. Sônia Renaux Wanderley Louro Departamento de Física - PUC-Rio

Prof. Hermi Felinto de Brito USP

Profa. Maria Luiza Rocco Duarte Pereira

UFRJ

Profa. Maria José Valenzuela Bell UFJF

Prof. José Eugênio Leal

Coordenador Setorial do Centro Técnico Científico - PUC-Rio 
Todos os direitos reservados. É proibida a reprodução total ou parcial do trabalho sem autorização da universidade, do autor e do orientador.

Welber Gianini Quirino

Graduou-se em Física pela Universidade Estadual Paulista Júlio de Mesquita Filho (UNESP), Bauru, São Paulo, em 2000. Obteve o título de Mestre em Física pela Universidade Federal de Juiz de Fora (UFJF), Juiz de Fora, Minas Gerais, em 2003.

Ficha Catalográfica

Quirino, Welber Gianini

Produção e caracterização de dispositivos orgânicos eletroluminescentes (OLEDs) baseados em complexos $\beta$ dicetonatos de Terras-Raras/ Welber Gianini Quirino; Orientador: Marco Cremona. - Rio de Janeiro: PUC, Departamento de Física, 2007.

1v., 260 p.: il.; $30 \mathrm{~cm}$

1. Tese (doutorado) - Pontifícia Universidade Católica do Rio de Janeiro, Departamento de Física.

Inclui referências bibliográficas.

1. Física - Teses. 2. Dispositivos eletroluminescentes. 3. OLEDs. 4. Eletroluminescência. 5. Complexos $\beta-$ dicetonatos de Terras-Raras. 6 . Filmes finos. 7. Nanotecnologia. I. Cremona, Marco. II. Pontifícia Universidade católica do Rio de Janeiro. Departamento de Física. III. Título.

CDD: 530 


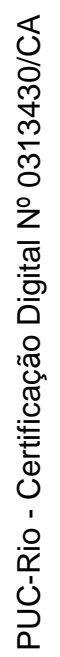

Mos meus fithos. Matheus e. Fuilia e à minha esposa Mariana. 


\section{Agradecimentos}

Agradeço a Deus.

Aos meus pais Valmir e Maria Helena pelo poio e incentivo constante.

Aos meus filhos Matheus e Júlia e à Minha esposa Mariana pelo carinho, pelo incentivo e pela paciência durante todos esses anos.

Aos meus familiares, Alda, René, Henrique, Daniel e Carolina, por toda ajuda durante este período.

Ao meu orientador, Marco Cremona, por ter me dado a oportunidade de concluir mais esta etapa, por ter tido interesse e dedicação em todos os momentos desse curso, por ter me ajudado nos momentos difíceis, além de ter me proporcionado um trabalho novo, empolgante e bonito.

Aos professores e técnicos do Departamento de Física, pelos conhecimentos adquiridos e pelo apoio técnico.

Aos Professores Hermi F. Brito, Marian Davolos, Severino A. Júnior e Petrus A. Santa Cruz pelos complexos orgânicos utilizados neste trabalho.

Aos colaboradores e amigos Ercules E. Teotônio, Marco A. Guedes, Patrícia Lima, Renata Adati, Sérgio Lima, Patrícia Nobrega, pelos complexos orgânicos sintetizados, pelos trabalhos e discussões em conjunto e pelo apoio em todos esses anos.

Aos meus amigos do Laboratório de Optoeletrônica Molecular (LOEM) Reynaldo, Cristiano, Sully, Paula, Letícia, Bernardo, Mariana, Laura, Renata, Denis, Rafael Brasil, Rafael Mendes, Denis, Helder e Felipe pelo apoio, sugestões, discussões e trabalhos desenvolvidos em conjunto. 
A todos os professores e funcionários da PUC-Rio que de maneira direta ou indireta contribuíram para a realização deste trabalho.

A Pontifícia Universidade Católica de Rio de Janeiro (PUC-Rio) por proporcionar as condições necessárias para o bom andamento do trabalho.

Ao Conselho Nacional de Desenvolvimento Científico e Tecnológico (CNPq), à Coordenação de Aperfeiçoamento de Pessoal de Nível Superior (Capes) e à Rede de Nanotecnologia Molecular e de Interfaces (Renami), pelo suporte financeiro. 


\section{Sumário}

1 Introdução 28

1.1. Estado da Arte 29

1.2. Justificativa 34

1.3. Escolha dos materiais $\quad 35$

1.4. Objetivos Gerais $\quad 35$

1.4.1. Objetivos Específicos $\quad 36$

1.5. Metas e Resultados Esperados 38

1.6. Organização da Tese 38

2 Dispositivos Orgânicos Emissores de Luz - OLEDs 42

2.1. Configuração e Funcionamento 42

2.1.1. Fabricação e Arquiterura dos Dispositivos 42

2.1.2. Funcionamento 45

2.2. Materiais $\quad 47$

2.2.1. Injetores de Carga 47

2.2.1.1. Injetores de Elétrons 48

2.2.1.2. Injetores de Buracos 50

2.2.2. Transportadores de Carga 53

2.2.2.1. Transportadores de Elétrons 53

2.2.2.2. Transportadores de Buracos 55

2.3. Mecanismos de Injeção e de Transporte de Cargas 56

2.3.1. Mecanismo de Injeção de Cargas em OLEDs 57

2.3.2. Influência do processo de transporte na condutividade elétrica 59

2.3.3. Injeção e transporte de cargas num OLED tipo heterojunção 61

2.3.4. A eletroluminescência nos OLEDs de heterojunção 65

2.3.5. Limites Operacionais de Funcionamento 67

2.3.5.1. Acoplamento Óptico 67

2.3.5.2. Eficiência Quântica 68

3 Os Íons Terras-Raras $\quad 75$

3.1. Introdução 75

3.2. Resumo Histórico 75 
3.3. Propriedades Gerais $\quad 76$

3.4. Propriedades Espectroscópicas dos Íons TR ${ }^{3+} \quad 77$

3.5. Os Complexos de Terras-Raras 80

3.5.1. Estrutura dos complexos $\beta$-dicetonatos de Terras-

Raras 80

3.5.2. Mecanismo de transferência de energia 81

3.5.3. Eletroluminescência dos complexos contendo íons lantanídeos $\quad 84$

4 Procedimentos Experimentais $\quad 90$

4.1. Introdução 90

4.2. Amostras 90

4.3. Síntese dos compostos $\beta$-dicetonatos de Terras-Raras 92

4.4. Preparação das amostras 93

4.4.1. Preparação dos substratos dos dispositivos 93

4.4.1.1. Padrões simples com fita adesiva 93

4.4.1.2. Padrões Especiais com Fotolitografia 96

4.4.1.3. Limpeza 99

4.5. Deposição de filmes finos 99

4.5.1. Deposição física de filmes finos por fase vapor 99

4.5.2. Descrição do sistema de deposição 101

4.5.3. Parâmetros utilizados para a deposição dos filmes

4.5.3.1. Parâmetros de calibração do sistema de medidas de espessura dos filmes orgânicos (INFICON) 104

4.5.4. Deposição de filmes finos por spin-coating 105

4.5.5. Desenho dos dispositivos 106

4.5.6. Fabricação dos OLEDs 108

5 Técnicas Analíticas $\quad 110$

5.1. Introdução 110

5.2. Medidas Ópticas $\quad 110$

5.2.1. Medidas de Fotoluminescência e Eletroluminescência 114

5.2.2. Absorbância 116

5.2.3. Medidas de Fotometria e Radiometria 117

5.2.3.1. Fluxo ou Potência Radiante 117 
5.2.3.2. Curva estandar de visibilidade do CIE 118

5.2.3.3. Fluxo Luminoso 118

5.2.3.4. Intensidade Radiante e Luminosa 120

5.2.3.5. Irradiância e lluminância 121

5.2.3.6. Radiância e Luminância 121

5.3. Medidas Elétricas 123

5.3.1. Medidas I vs V 124

5.4. Caracterização Física e Química das amostras 125

5.4.1. Medidas de Espessura de Filmes Finos 125

5.4.2. Voltametria Cíclica 126

5.4.2.1. Obtenção dos parâmetros eletroquímicos $\quad 126$

5.4.2.2. Eletrodos quimicamente modificados $\quad 130$

5.4.2.3. Medidas de Voltametria Cíclica 131

5.4.3. Transição Vítrea 133

6 Fabricação e Caracterização de OLEDs usando complexos $\beta$-dicetonatos de Terras-Raras como camada emissora 136

6.1. Introdução 136

6.2. Sistema 1 - O Complexo $\left[\mathrm{Eu}(\mathrm{bmdm})_{3}(\mathrm{ttpo})_{2}\right] \quad 136$

6.2.1. Experimental 137

6.2.1.1. Síntese do Complexo 137

6.2.1.2. Fabricação dos dispositivos 138

6.2.2. Resultados e Discussão 139

6.2.3. Conclusão (Sistema 1) 157

6.3. Sistema 2 - O Complexo Binuclear $\beta$-dicetonato de Eu e Tb 159

6.3.1. Introdução 159

6.3.1.1. Sistema Aceitador-Doador 161

6.3.2. Experimental 161

6.3.2.1. Síntese do Complexo binuclear 162

6.3.2.2. Fabricação dos Dispositivos 163

6.3.3. Resultados e Discussão 164

6.3.4. Conclusão (Sistema 2) 188

6.4. Sistema 3 - Os Complexos tetrakis( $\beta$-dicetonatos) de $\mathrm{TR}^{3+}$ -

(Sais de Lítio) 190

6.4.1. Introdução 190

6.4.2. Experimental 191 
6.4.2.1. Os Complexos de Eu e Tb

6.4.2.2. Fabricação dos Dispositivos 192

6.4.3. Resultados e Discussão 193

6.4.3.1. Caracterizações ópticas dos compostos dos sais de lítio na forma de pó e filmes finos 193

6.4.3.2. Dispositivos com o complexo Li[Eu(dbm) $)_{4} \quad 197$

6.4.3.3. Dispositivos com complexo Li[Tb(acac) $\left.)_{4}\right] 202$

6.4.4. Conclusão (Sistema 3) 208

7 Fotodegradação de Compostos Orgânicos 212

7.1. Introdução 212

7.2. Espectroscopia de fotoelétrons 213

7.2.1. Espectroscopia de Fotoelétrons de Raios X (XPS - Xray Photoelectron Spectroscopy) 214

7.3. Espectroscopia de Fotoabsorção - NEXAFS (Near Edge X-ray Absorption Fine Structure) 216

7.3.1. Ressonâncias $\pi^{*} \quad 218$

7.3.2. Ressonâncias de Forma, $\sigma^{*} \quad 218$

7.4. Luz Síncrotron $\quad 219$

7.4.1. Linhas de Luz utilizadas neste trabalho 221

7.4.2. D08A - SGM (Spherical Grating Monochromator) 221

7.4.3. D05A - TGM (Toroidal Grating Monochromator) 221

7.5. Experimental 222

7.5.1. Deposição dos filmes 222

7.5.2. Montagem dos filmes orgânicos para análise por UPS,

XPS e NEXAFS 222

7.6. Resultados 224

7.6.1. Alq $_{3}$

7.6.2. MTCD 230

7.6.3. Complexo de Európio - Eu(TTA $)_{3}(\text { TPPO })_{2}$

7.6.4. Conclusão 240

7.7. Aplicação - OLED usado como dosímetro portátil de radiação UV 242

7.7.1. Introdução 242

7.7.2. Experimental $\quad 244$

7.7.3. Resultados $\quad 245$ 
8 Conclusões

9 Produção

259

9.1. - Lista de publicações

259

9.2. - Patente registrada

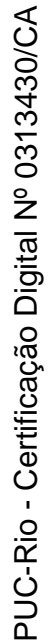




\section{Lista de Figuras}

Fig. 2.1 - Arquiteturas típicas de OLEDs - (a) OLED monocamada, (b) OLED bicamada, (c) OLED tricamada e (d) OLED multicamada, onde CIB é a camada injetora de buracos, CTB é a camada transportadora de buracos, CEL é a camada eletroluminescente, CTE a camada transportadora de electrons e CIE a camada injetora de elétrons.

Fig. 2.2 - Representação da região de recombinação. (a) No dispositivo monocamada as mobilidades entre buracos e elétrons são bem diferentes, causando a recombinação em várias regiões diferentes, sendo que muitas das cargas são perdidas ao se recombinarem perto dos eletrodos. (b) no dispositivo tri-camada ideal as mobilidades de cargas são balanceadas, fazendo com que os portadores se recombinem numa região ótima, ou seja, dentro da camada CEL. ........................................................................ 45

Fig. 2.3 - Processo esquemático da Eletroluminescência. ................................46

Fig. 2.4 - Diagrama de energias para um OLED monocamada deAlq ${ }_{3}$ para dois metais, mostrando a diferença entre $\mathrm{Mg}$ e Al. 48

Fig. 2.5 - Característica (corrente-voltagem) para três OLEDs usando Al, Mg:Ag e LiF/Al como catodo, respectivamente [3] 49

Fig. 2.6 - (I a IV) Representação de algumas das várias reflexões que a luz emitida por um OLED pode sofrer ao passar de uma camada para outra até sair através do vidro. Também há emissão de luz pelas laterais do dispositivo (V), mas como a espessura de todas as camadas somadas não passa de algumas centenas de nanômetros, essa porção de luz é praticamente desprezível. Dependendo dos índice de refração das várias camadas e também do ângulo em que raio de luz é emitido dentro da zona de recombinação, este pode sofrer reflexões totais e/ou parciais durante sua trajetória até escapar para o ambiente. Nota: Usaremos na seção 2.3.5 essa mesma figura para analisar mais detalhadamente os limites operacionais de funcionamento. 53

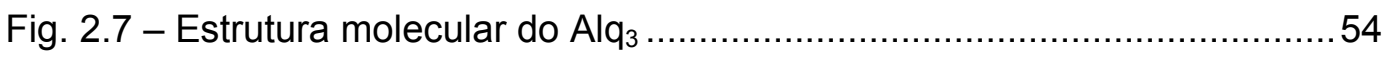

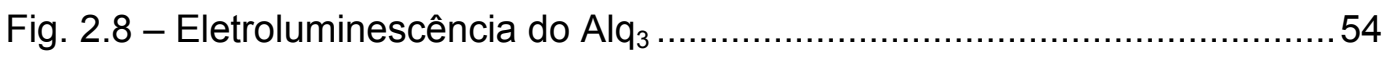

Fig. 2.9 - Estrutura molecular de materiais orgânicos transportadores de buracos

Fig. 2.10 - Representação da injeção por tunelamento tipo Fowler-Nordheim e a injeção termoiônica, em uma estrutura metal/semicondutor-intrínseco/metal. 
$\varphi$ é a altura da barreira de potencial na interface metal/semicondutor e $V$ é o potencial elétrico aplicado entre os eletrodos........................................57

Fig. 2.11 - (a) Vista em seção esquemática de um OLED com estrutura tipo heterojunção. (b) Esquema mostrando a distribuição exponencial de armadilhas no $\mathrm{Alq}_{3}$, em relação às energias orbitais moleculares e o quasenível eletrônico de Fermi. (HOMO: highest occupied molecular orbital; LUMO: lowest unoccupied molecular orbital).

Fig. 2.12 - Junção orgânica "tipo p-n" num dispositivo bicamanda sob tensão. A recombinação na CEL emite luz verde $(\sim 510 \mathrm{~nm})$ proveniente dos estados excitados do $\mathrm{Alq}_{3}$.

Fig. 2.13 - Esquema mostrando a relaxação dos elétrons aprisionados no $\mathrm{Alq}_{3}$ com um buraco minoritário, para formar uma distribuição de éxcitons Frenkel no gap do $\mathrm{Alq}_{3}$, os quais posteriormente se recombinam para gerar a emissão eletroluminescente.

Fig. 2.14 - Modelo clássico para a distribuição da luz no interior de um dispositivo OLED. 68

Fig. 2.15 - Diagrama esquemático mostrando os processos de Eletroluminescência.

Fig. 3.1 - Níveis de energia para os $\mathrm{TR}^{3+}$. Os níveis são obtidos por experimento de absorção no qual é incidida sobre o íon no estado fundamental radiação, cuja freqüência é variada continuamente $[$. 79

Fig. 3.2 - Estrutura simplificada dos complexos de terras-raras octacoordenados. [O] indica o íon terra-rara central, [•] indica os átomos de oxigênio e/ou nitrogênio provenientes dos ligantes. 81

Fig. 3.3 - (a) Esquema do mecanismo de transferência de energia e emissão dos complexos de terras-raras. Como exemplo foi usada uma das molécula $\beta$ dicetonas estudadas nesta tese $\left(\mathrm{Eu}(\mathrm{dmbm})_{3}(\mathrm{ttpo})_{2}\right)$, que será descrita posteriormente. (b) Esquema ilustrativo dos possíveis processos de transferência de energia de um ligante orgânico para um íon lantanídeo trivalente, $\mathrm{Ln}^{3+}$, neste exemplo específico o íon $\mathrm{Eu}^{3+}$, onde o nível ${ }^{5} \mathrm{D}_{1}$ é mais baixo que o estado tripleto $T_{1}$. Notação: $A=$ absorção; $F=$ fluorescência; $P$ = fosforescência; $\mathrm{Cl}$, conversão interna; $\mathrm{W}=$ relaxação cruzada interna e TE = transferência de energia

Fig. 3.4 - Representação esquemática do processo de emissão eletroluminescente envolvendo o "efeito antena". .86

4.1 - Esquema do desenvolvimento do processo de Fotolitografia. .97 
Fig. 4.2 - Fotos mostrando o equipamento de deposição utilizado (UNIVEX 300) (esquerda) e parte interior da câmara de alto vácuo, onde são colocados os cadinhos com os materiais a serem depositados. 101

Fig. 4.3 - Esquema simplificado do sistema de deposição térmico resistivo utilizado para a deposição dos filmes orgânicos. (1) câmara de deposição, (2) visor (obló), (3) eixo central para rotação do porta-amostra, (4) portaamostra, (5) "shutter", (6) cristal de quartzo do controlador XTC-INFICON, (7) cadinhos, (8) eletrodos da fonte de alimentação. 102

Fig. 4.4 - porta substratos usado na deposição dos filmes e dispositivos orgânicos. 103

Fig. 5.1 - Diagrama ilustrando a absorção, o decaimento radiativo e não radiativo caracterizados pelas suas taxas $\Gamma$ e k, respectivamente. (A, absorção; F, fluorescência).

Fig. 5.2 - Diagrama esquemático do espectrofluorímetro PTI modelo 1469. O sistema eletrônico de controle e o computador não são mostrados.

Fig. 5.3 - Diagrama esquemático do espectrofluorímetro PTI modelo 1469. A região em destaque ilustra como é feita a conexão para a medida de eletroluminescência.

Fig. 5.4 - Exemplo de um espectro de absorção de um material na vizinhança do edge $\left(\lambda_{0}\right)$ de absorção. Nesta aproximação, $\mathrm{Eg}=\mathrm{hc} / \lambda_{0}$.

Fig. 5.5 - Função da luminosidade relativa, como definido pela CIE para a visão fotóptica normal.

Fig. 5.6 - Esquema utilizado nesta tese para obter as curvas características I-V dos OLEDs. $(\Omega)$ resistência elétrica $(1 \mathrm{~K} \Omega)$.

Fig. 5.7 - Linha traçada pelo perfilômetro de um risco feito num filme orgânico.

Fig. 5.8 - Diagrama de bandas para uma estrutura metal/orgânico/metal. 126

Fig. 5.9 - Relação entre o potencial eletroquímico, E'ox e E'red, potencial de ionização IP e banda proibida Eg, de um estrutura de bandas de uma molécula.

Fig. 5.10 - Voltamograma obtido para a determinação do potencial de ionização. Em destaque, o ponto onde se determina o IP. 130

Fig. 5.11 - Ciclovoltamograma de um filme fino de $\mathrm{Alq}_{3}$ depositado sobre o eletrodo de grafite.

Fig. 6.1 - Estrura química do ligante bmdm. 136

Fig. 6.2 - Representação esquemática do complexo $\left[\mathrm{Eu}(\mathrm{bmdm})_{3}(\mathrm{tppo})_{2}\right]$ 138 
Fig. 6.3 - Espectros de Absorção na região do UV-vis do complexo de Európio (linha pontilhada - gap $=3,22 \mathrm{eV}$ ), bmdm (linha tracejada - gap $=3,22 \mathrm{eV}$ ) e tppo (linha contínua - gap $=4,50 \mathrm{eV}$ ) todos em solução alcoólica e medidos a temperatura ambiente. 140

Fig. 6.4 - Espectro de fotoluminescência a $77 \mathrm{~K}$ do complexo de Európio, mostrando as estreitas e típicas linhas de emissão do íon $\mathrm{Eu}^{3+}$, atribuídas às transições ${ }^{5} D_{0}-{ }^{7} F_{J}(J=0,1,2,3,4)$. O gráfico inserido mostra o espectro de fotoluminescência à temperatura ambiente de um filme de $50 \mathrm{~nm}$ depositado termicamente sobre quartzo. 141

Fig. 6.5 - Estrutura esquemática do dispositivo 1 142

Fig. 6.6 - Eletroluminescência do dispositivo 1 - (bicamada) em função da tensão aplicada. O gráfico inserido mostra o aumento da intensidade da transição hipersensível ${ }^{5} \mathrm{D}_{0^{-}}{ }^{7} \mathrm{~F}_{2}$ em função do aumento da tensão aplicada. 143

Fig. 6.7 - Diagrama de Cromaticidade $(X, Y)$ CIE para o dispositivo $1, X=0.66, Y$ $=0,33$ (circulo) 144

Fig. 6.8 - Característica I vs V para o dispositivo 1 - bicamada ....................... 144

Fig. 6.9 - Estrutura esquemática do dispositivo 2 145

Fig. 6.10 - Eletroluminescência do dispositivo 2 - tricamada em função da tensão aplicada. 146

Fig. 6.11 - A transição radiativa ${ }^{5} D_{1} \rightarrow{ }^{7} F_{1}$ não é favorecida devido ao efeito de desativação por relaxação cruzada entre íons TR vizinhos. 147

Fig. 6.12 - Espectro de Fotoluminescência do complexo ligante bmdm excitado a $\lambda=360 \mathrm{~nm}$. 148

Fig. 6.13 - Eletroluminescência do dispositivo 3 em função de algumas tensões aplicadas.

Fig. 6.14 - Eletroluminescência do dispositivo $3 \mathrm{com}$ o ligante bmdm como camada emissora (linha preta contínua). Ajuste gaussiano realizado na banda larga do dispositivo 2 (linha pontilhada vermelha) 149

Fig. 6.15 - Esquema ilustrativo dos possíveis processos de transferência de energia de um ligante orgânico para o íon lantanídeo trivalente, $\mathrm{Eu}^{3+}$, com ênfase para o mecanismo de eletrofosforescência molecular $(E P), T_{1}-S_{0}$, linha (3). Notação: $A=$ absorção; $F=$ fluorescência; $E P=$ Eletrofosforescência; $\mathrm{Cl}$, conversão interna; $\mathrm{W}$ = relaxação cruzada interna e TE = transferência de energia. 150

Fig. 6.16 - Diagrama de Cromaticidade $(X, Y)$ CIE para o dispositivo $1, X=0.66$, $Y=0,33$ (circulo) e para o dispositivo $2, X=0.46, Y=0.44$ (triângulo) ....151 
Fig. 6.17 - Eletroluminescência de outro dispositivo tricamada em função da tensão aplicada.

Fig. 6.18 - Característica I vs $\mathrm{V}$ do segundo dispositivo 2 - tricamada, utilizando a fonte de tensão programável Keithley 2240.

Fig. 6.19 - Característica I vs $\mathrm{V}$ do dispositivo 2 153

Fig. 6.20 - Comportamento característico para o gráfico $\ln \left(\mathrm{I} / \mathrm{V}^{2}\right) \times(1 / \mathrm{V})$ para 0 OLED baseado $\mathrm{Eu}(\mathrm{bmdm})_{3}(\mathrm{ttpo})_{2}$ tricamada. É possível distinguir duas regiões distintas de injeção de cargas; a primeira $(p)$ onde temos injeção predominante de buracos e uma segunda $(n)$ com injeção predominante de elétrons. As duas linhas contínuas representam aproximações do modelo de injeção por tunelamento (Fowler-Nordheim) 154

Fig. 6.21 - Voltamograma do complexo $\left[\mathrm{Eu}(\mathrm{bmdm})_{3}(\mathrm{tppo})_{2}\right]$. O complexo é depositado sobre o eletrodo de grafite. A expressão matemática inserida no gráfico mostra o cálculo realizado para achar o valor em energia do HOMO, utilizando a expressão (5.7) da Sec. (5.4.2). 155

Fig. 6.22 - Diagrama rígido de energia obtido por medidas de voltametria cíclica para determinar o HOMO dos compostos. O LUMO é obtido pela soma dos valores do HOMO com o gap óptico, obtido por medidas de absorbância. As linhas pontilhadas representam a posição do $\mathrm{Alq}_{3}(\mathrm{HOMO}=5,6 \mathrm{eV}$ e LUMO $=2,6 \mathrm{eV})$.

Fig. 6.23 - Passado e futuro da iluminação ambiente. Um OLED branco produzido pela GE Global Research, que produz 70 lúmens e com uma eficiência de $7 \mathrm{Im} / \mathrm{W}$. 160

Fig. 6.24 - Sistema doador-aceitador. A sobreposição das bandas é condição necessária para transferência de energia 161

Fig. 6.25 - Estrutura Molecular do complexo Eu(btfa) $)_{3} \bullet$ phenterpy $\bullet \operatorname{Tb}(\mathrm{acac})_{3} \ldots 162$ Fig. 6.26 - Estrutura molecular do ligante phenterpy - 4',4"''-(1,4-Phenileno)bis$\left(2,2^{\prime}: 6\right.$ ',2"-terpiridina), chamado ao longo deste trabalho de apenas "phenterpy". 163

Fig. 6.27 - Espectros de excitação na região do UV-vis do complexo binuclear, (linha tracejada vermelha - detector em $614 \mathrm{~nm},(\mathrm{Eu})$ ), (linha contínua detector em $440 \mathrm{~nm}$, (Tb)). Ambos os espectros foram obtidos a partir de um filme do complexo binuclear depositado termicamente sobre quartzo e medidos a temperatura ambiente. O gap óptico foi estimado em 3,61 eV.

Fig. $6.28-$ Espectros de Fotoluminescência do complexo $\mathrm{Eu}(\mathrm{btfa})_{3} \cdot$ phenterpy $\cdot \mathrm{Tb}(\mathrm{acac})_{3}$ (a) baixa temperatura e (b) temperatura 
ambiente com $\lambda$ exc $=340 \mathrm{~nm}$. O pico em $614 \mathrm{~nm}$ corresponde à transição eletrônica ${ }^{5} \mathrm{D}_{0} \rightarrow{ }^{7} \mathrm{~F}_{2}$ do $\mathrm{Eu}^{3+}$, enquanto que o pico em $550 \mathrm{~nm}$ corresponde à transição eletrônica do ${ }^{5} \mathrm{D}_{4} \rightarrow{ }^{7} \mathrm{~F}_{5}$ do $\mathrm{Tb}^{3+}$. 166

Fig. 6.29 - Espectro de Fotoluminescência à temperatura ambiente do filme de $\mathrm{Eu}(\mathrm{btfa})_{3} \cdot$ phenterpy $\cdot \mathrm{Tb}(\mathrm{acac})_{3}$ depositado sobre quartzo com $\lambda_{\mathrm{exc}}=340 \mathrm{~nm}$. Não há diferença significativa entre este espectro e o obtido a partir do composto em pó. 167

Fig. 6.30 - Estrutura esquemática do dispositivo 1 168

Fig. 6.31 - Espectros de Electroluminescência a temperatura ambiente do dispositivo 1: ITO/NPB(40)/[Eu(btfa) $)_{3}$ phenterpy•Tb(acac) $\left.)_{3}(20)\right] / \mathrm{Al}(100)$ para diferentes valores de voltagem. A banda larga centrada em $580 \mathrm{~nm}$ pode ser associada com a eletrofosforescência do ligante mais a eletroemissão do NPB. A seta indica a eletroemissão do Európio sob a banda da eletrofosforescência do ligante. O gráfico inserido mostra a característica I vs $\mathrm{V}$ de um diodo típico. 169

Fig. 6.32 - Foto do dipositivo 1 - ITO/ NPB(40)/ $\left[\mathrm{Eu}(\mathrm{btfa})_{3} \cdot\right.$ phenterpy• $\left.\mathrm{Tb}(\mathrm{acac})_{3}(20)\right] / \quad \mathrm{Al}(100)$ em funcionamento com emissão predominantemente azul, conseqüência da soma das emissões: (i) fluorescência do NPB, (ii) eletrofosforescência dos ligantes. 170

Fig. 6.33 - Diagrama de Cromaticidade $(X, Y)$ CIE para o dispositivo $1, X=0,24$; $\mathrm{Y}=0,19$ (círculo) 170

Fig. 6.34 - Espectros de electroluminescência à temperatura ambiente do dispositivo 2: ITO/NPB(40)/phenterpy(20)]/AI(100) para diferentes valores de voltagem. A banda larga centrada em $510 \mathrm{~nm}$ pode ser associada com a eletrofosforescência do ligante phterpy. 171

Fig. 6.35 - Espectros de electroluminescência na região do azul à temperatura ambiente do dispositivo 2: ITO/NPB(40)/AI(100) para diferentes valores de tensão.

Fig. 6.36 - Eletroluminescência do dispositivo 1 com o complexo binuclear como camada emissora (linha tracejada azul); Eletroluminescência do dispositivo 3 com o complexo phterpy como camada emissora (linha pontilhada preta). Ajuste gaussiano realizado na banda larga do dispositivo 3 (linha vermelha contínua).

Fig. 6.37 - Diagrama de Cromaticidade $(X, Y)$ CIE para o dispositivo 1, $X=0,15$, $\mathrm{Y}=0,12$ (círculo).

Fig. 6.38 - Característica I vs V para o dispositivo 2. 174

Fig. 6.39 - Característica I vs $\vee$ do segundo dispositivo 2 com eixos log-log....175 
Fig. 6.40 - Estrutura esquemática do dispositivo 4 176

Fig. 6.41 - Espectros de electroluminescência à temperatura ambiente do dispositivo 4: ITO/NPB $(40) /\left[\mathrm{Eu}(\mathrm{btfa})_{3} \cdot\right.$ phenterpy• $\left.\mathrm{Tb}(\mathrm{acac})_{3}(20)\right] / \mathrm{Alq}_{3}(30) / \mathrm{Al}(100) \quad$ para diferentes valores de voltagem. Houve um aumento da banda larga centrada em $580 \mathrm{~nm}$ associada com a eletrofosforescência do ligante. Este aumento se deve ao aumento da banda centrada em $510 \mathrm{~nm}$ associada com a emissão do $\mathrm{Alq}_{3}$. A seta indica a eletroeluminescência do Európio sob a banda da eletrofosforescência do ligante. 177

Fig. 6.42 - Foto do dipositivo 4 em funcionamento. A intensa emissão branca (20 $\mathrm{cd} / \mathrm{m}^{2}$ ) é conseqüência da soma das emissões: (i) fluorescência do NPB, (ii) eletrofosforescência do ligante, (iii) fluorescência do $\mathrm{Alq}_{3}$ e (iv) fluorescência do $\mathrm{Eu}^{3+}$

Fig. 6.43 - Espectros de Electroluminescência à temperatura ambiente do dispositivo 5 com maoir espessura do complexo binuclear $(40 \mathrm{~nm})$ e menor espessura do $\mathrm{Alq}_{3}(20 \mathrm{~nm})$ para diferentes valores de voltagem aplicada.

Fig. 6.44 - Diagrama de Cromaticidade $(X, Y)$ CIE (círculo) para o dispositivo 4 funcionando a $18 \mathrm{~V}$ e (triângulos) quando o dispositivos está funcionando a diferentes voltagens $(A=23 \mathrm{~V}, B=25 \mathrm{~V}, \mathrm{C}=27 \mathrm{~V}, \mathrm{D}=28 \mathrm{~V}$ and $\mathrm{E}=30)$. $\mathrm{O}$ espectro correspondente a cada um desses pontos está mostrado na Fig. 6.43 ...179

Fig. 6.45 - Voltamograma do complexo binuclear. O complexo é depositado sobre o eletrodo de grafite por meio de uma solução alcoólica. 181

Fig. 6.46 - Diagrama de energia obtido por medidas de voltametria cíclica para determinar o HOMO dos compostos NPB, binuclear e $\mathrm{Alq}_{3}$. O LUMO é obtido pela soma dos valores do HOMO com o gap óptico, obtido por medidas de absorbância. As linhas pontilhadas representam a posição do $\mathrm{Alq}_{3}(\mathrm{HOMO}=5,6 \mathrm{eV}$ e LUMO = 2,6 eV $)$.

Fig. 6.47 - Estrutura molecular do ligante 4'4'Bipiridina. 182

Fig. 6.48 - Espectro de Fotoluminescência do $\mathrm{Eu}(\mathrm{btfa})_{3} \cdot \mathrm{Bipy} \bullet \mathrm{Tb}(\mathrm{acac})_{3}$ à temperatura ambiente com $\lambda=357 \mathrm{~nm}$ e $\lambda=369,5 \mathrm{~nm}$. O pico em $614 \mathrm{~nm}$ corresponde à transição eletrônica ${ }^{5} \mathrm{D}_{0} \rightarrow{ }^{7} \mathrm{~F}_{2}$ do $\mathrm{Eu}^{3+}$. O gráfico inserido é uma ampliação da região 450 a 560 e mostra os picos característicos do $\mathrm{Tb}^{3+}$, onde a transição em $550 \mathrm{~nm}$ corresponde à transição eletrônica ${ }^{5} \mathrm{D}_{4} \rightarrow$ ${ }^{7} \mathrm{~F}_{5}$

Fig. 6.49 - Espectros de excitação do complexo binuclear 2. Curva de excitação obtida com o detector centrado na transição hipersensível do $\mathrm{Eu}^{3+}$ (linha 
vermelha contínua). Curva de excitação obtida com o detector centrado na transição hipersensível do $\mathrm{Tb}^{3+}$ (linha tracejada verde). 184

Fig. 6.50 - Espectros de electroluminescência à temperatura ambiente do dispositivo A: ITO/NPB(40)/[Eu(btfa) $\left.)_{3} \cdot \mathrm{Bipy} \cdot \mathrm{Tb}(\mathrm{acac})_{3}(10)\right] / \mathrm{Alq}_{3}(30) / \mathrm{Al}(100)$ para diferentes valores de voltagem. Neste caso, uma banda larga está cobrindo todo o espectro de 400 a $700 \mathrm{~nm}$. Esta banda está, como no caso dos dispositivos anteriores, associada com a eletrofosforescência do ligante mais a eletroemissão do NPB e do $\mathrm{Alq}_{3}$. É possível identificar agora a eletroemissão do $\mathrm{Tb}^{3+}$ junto com algumas bandas do $\mathrm{Eu}^{3+}$, ambas parcialmente encobertas pela banda larga mencionada acima................. 185

Fig. 6.51 - Característica I vs $\vee$ do dispositivo 6 - binuclear 2 ..................... 186

Fig. 6.52 - Estrutura molecular do ligante bzac = 1-fenil-1,3-butanodiona ....... 186

Fig. 6.53 - Espectros de Electroluminescência a temperatura ambiente do OLED baseado no composto trinuclear, com $\operatorname{Tm}(0,8), \operatorname{Tb}(0,15)$ e $\operatorname{Eu}(0,05)$. O resultado é uma banda larga que cobre todo o espectro visível.

Fig. 6.54 - Fórmula estrutural do complexo tetrakis-dibenzoilmetrano de európio (III) tetrahidratado. 192

Fig. 6.55 - Fórmula estrutural do complexo tetrakis-acetilacetonato de térbio (III). 192

Fig. 6.56 - O espectro de fotoluminescência do complexo sólido $\mathrm{Li}\left[\mathrm{Eu}(\mathrm{dbm})_{4}\right.$ à baixa temperatura com $\lambda_{\mathrm{exc}}=394 \mathrm{~nm}$ mostra as transições típicas do íon $\mathrm{Eu}^{3+}\left({ }^{5} \mathrm{D}_{0}{ }^{-7} \mathrm{~F}_{0,1,2,3,4}\right)$, acima. $\mathrm{O}$ espectro de fotoluminescência do complexo sólido $\mathrm{Li}\left[\mathrm{Tb}(\mathrm{acac})_{4}\right.$ à baixa temperatura com $\lambda_{\mathrm{exc}}=342 \mathrm{~nm}$ mostra as transições típicas do íon $\mathrm{Tb}^{3+}\left({ }^{5} \mathrm{D}_{4}{ }^{7} \mathrm{~F}_{2,3,4,5,6}\right)$, abaixo. 195

Fig. 6.57 - Espectros de absorção e fotoluminescência na região do UV-vis do filme do compelxo $\mathrm{Li}\left[\mathrm{Tb}(\mathrm{acac})_{4}\right]$, gráfico (a). Espectros de absorção, excitação e de fotoluminescência do filme de $\mathrm{Li}\left[\mathrm{Eu}(\mathrm{dbm})_{4}\right]$, gráfico (b). Todos os espectros foram obtidos a partir de um filme fino sobre uma amostra de quartzo a temperatura ambiente......................................... 197

Fig. 6.58 - Estrutura esquemática do dispositivo 1 ..................................... 198

Fig. 6.59 - Espectros de electroluminescência à temperatura ambiente do dispositivo 1: ITO/NPB(40)/Li[Eu(BDM) $\left.)_{4}\right](50) / \mathrm{Al}(160)$ para diferentes valores de voltagem. A pequena banda larga centrada em $435 \mathrm{~nm}$ pode ser associada com a eletroemissão do NPB. O gráfico inserido mostra que o dispositivo funciona como um diodo típico. 200

Fig. 6.60 - Espectros de electroluminescência à temperatura ambiente do dispositivo 1: ITO/NPB(40)/Li[Eu(BDM) 4 (50)/Alq3(25)/Al(130) para 
diferentes valores de voltagem. A banda larga centrada em $435 \mathrm{~nm}$ é devida à eletroemissão do NPB. O gráfico inserido, mostra que o dispositivo funciona como um diodo típico. 200

Fig. 6.61 - Espectros de electroluminescência à temperatura ambiente do dispositivo 3: ITO/NPB(40)/Li[Eu(BDM)4](70)/LiF(0,5)/AI(150) para diferentes valores de voltagem. 201

Fig. 6.62 - Foto do dipositivo 3 em funcionamento com emissão predominantemente vermelha do íon Európio 202

Fig. 6.63 - Diagrama de Cromaticidade $(X, Y)$ CIE para o dispositivo $1, X=0,54$, $Y=0,35$, dispositivo $2, X=0,40 ; Y=0,33$ e dispositivo $3, X=0,56 ; Y=0,36$.

Fig. 6.64 - Espectros de Electroluminescência a temperatura ambiente do dispositivo 4: ITO/NPB(40)/Li[Tb(acac) 4$](50) / \mathrm{Al}(160)$ para diferentes valores de voltagem. A banda larga centrada em $435 \mathrm{~nm}$ é devida à eletroemissão do NPB. 203

Fig. 6.65 - Espectros de electroluminescência à temperatura ambiente do dispositivo 5: ITO/MTCD $(40) / \mathrm{Li}\left[\mathrm{Tb}(\mathrm{acac})_{4}\right](50) / \mathrm{Al}(160)$ para diferentes valores de voltagem. 204

Fig. 6.66 - Diagrama de Cromaticidade $(X, Y)$ CIE para o dispositivo 4, $X=0,22$, $\mathrm{Y}=0,43$ e para o dispositivo $5, \mathrm{X}=0,24 ; \mathrm{Y}=0,50$. 205

Fig. 6.67 - Característica I vs $\vee$ para o dispositivo 5 - bicamada 205

Fig. 6.68 - Voltamogramas dos sais de lítio. O complexo é depositado sobre o eletrodo de grafite por meio de uma solução alcoólica 206

Fig. 6.69 - Diagrama rígido de energia obtido por medidas de voltametria cíclica para determinar o HOMO dos compostos NPB, binuclear e $\mathrm{Alq}_{3}$. O LUMO é obtido pela soma dos valores do HOMO com o gap óptico, obtido por medidas de absorbância. As linhas pontilhadas representam a posição do $\mathrm{Alq}_{3}(\mathrm{HOMO}=5,6 \mathrm{eV}$ e LUMO = 2,6 eV). .....................................208

Fig. 7.1 - Esquema do processo de fotoemissão. ........................................215

Fig. 7.2 - Mapa das linhas de Luz instaladas no LNLS. As linhas de luz que operam em raio-x estão representadas em azul, as linhas de luz que operam em ultravioleta e raios-x moles estão representadas em vermelho. Também aparecem no mapa, em verde, as três novas linhas previstas...220 Fig. 7.3 - Disposição dos filmes dos compostos orgânicos empregados em OLEDs no porta-amostra utilizado para os experimentos de UPS, XPS e NEXAFS realizados no LNLS 223 
Fig. 7.4 - Representação 3D dos espectros de emissão (FL) do MTCD - gráfico (a), do $\mathrm{Alq}_{3}$ - gráfico (b) e do complexo de Európio - gráfico (c), irradiados com lâmpada UV em 379, 397 e 348 nm, respectivamente. Decaimento da intensidade de $\mathrm{FL}$ em função do tempo de irradiação para os três compostos, gráfico (d). As constantes de tempo para os compostos são 45,8 \pm 1,8 min para o MTCD, 19,6 \pm 1,4 min para o $\mathrm{Alq}_{3}$ e 23,2 $\pm 2,1 \mathrm{~min}$ para o complexo $\left[\mathrm{Eu}(\mathrm{TTA})_{3}(\mathrm{TPPO})_{2}\right]$, considerando-se a transição

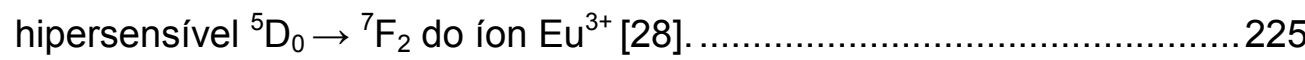

Fig. 7.5 - Espectro de NEXAFS na borda 1s do carbono do $\mathrm{Alq}_{3}$ degradado (linha vermelha), em comparação com o do filme não-degradado (linha preta). 227

Fig. 7.6 - Orbitais moleculares do $\mathrm{Alq}_{3}$ obtidos pelo programa computacional Gaussian 98, utilizando o método Hartree-Fock na base 6-311g*: a) HOMO, b) LUMO, c) LUMO+1, d) LUMO+2 ${ }^{[16]}$ 228

Fig. 7.7 - Espectro de NEXAFS na borda 1s do nitrogênio do $\mathrm{Alq}_{3}$ (degradado e não-degradado). 229

Fig. 7.8 - Espectro de NEXAFS na borda 1s do oxigênio do $\mathrm{Alq}_{3}$ degradado (linha vermelha), em comparação com o do filme não-degradado (linha preta). 230

Fig. 7.9 - Espectro de NEXAFS na borda $K$ do nitrogênio do MTCD degradado (linha vermelha), em comparação com o do filme não-degradado (linha preta). 231

Fig. 7.10 - Espectro de fotoemissão do MTCD na borda 1s do nitrogênio, antes (linha preta) e depois da irradiação em ordem zero da luz síncrotron (linha vermelha) $\left(\sim 10^{10}\right.$ fótons $\left.\mathrm{s}^{-1} \mathrm{~cm}^{-2}\right)$. 232

Fig. 7.11 - Espectros de fotoemissão e de fotodegradação do MTCD na borda 1s do carbono ( $h v=400 \mathrm{eV})$, antes (linha preta) e depois da exposição à luz branca (ordem zero) da luz síncrotron $\left(\sim 10^{10}\right.$ fótons $\left.\mathrm{s}^{-1} \mathrm{~cm}^{-2}\right)$. 233

Fig. 7.12 - Espectros de fotoemissão do MTCD cobrindo a banda de valência $(\mathrm{h} v=250 \mathrm{eV})$ antes (linha preta) e depois de 20 minutos de exposição à luz branca (ordem zero) da luz síncrotron (linha vermelha)..........................234

Fig. 7.13 - Estrutura molecular do complexo de Európio - Eu(TTA) $)_{3}(\text { TPPO })_{2}$.. 235

Fig. 7.14 - Espectro de NEXAFS na borda 1s do carbono e do oxigênio do complexo de Európio $\mathrm{Eu}(\mathrm{TTA})_{3}(\mathrm{TPPO})_{2}$ degradado (linha vermelha), em comparação com o do filme não-degradado (linha preta). 235

Fig. 7.15 - Espectros de fotoemissão e fotodegradação do complexo de Európio cobrindo da banda de valência $(\mathrm{h} v=250 \mathrm{eV}$ ) até a borda 1s do carbono ( $\mathrm{h} v$ $=400 \mathrm{eV}$ ) antes (linha preta) e depois de 30 minutos de exposição à luz branca (ordem zero) da luz síncrotron (linha vermelha). 
Fig. 7.16 - Espectros de fotoemissão e fotodegradação do complexo de Európio cobrindo da borda do Eu $4 \mathrm{p}(\mathrm{h} v=400 \mathrm{eV})$ até a borda 1s do oxigênio ( $\mathrm{h} v=$ $600 \mathrm{eV}$ ) antes (linha preta) e depois de 30 minutos de exposição à luz branca (ordem zero) da luz síncrotron (linha vermelha)..........................239

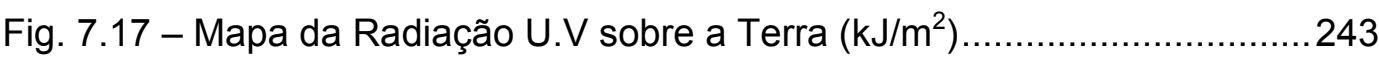

Fig. 7.18 - Estrutura esquemática do dispositivo tricamada usado como

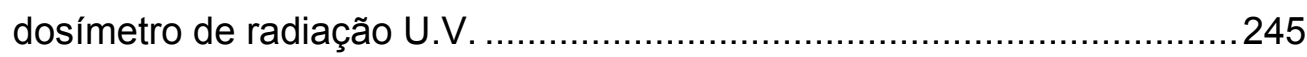

Fig. 7.19 - Estrutura molecular do complexo Eu(btfa) ${ }_{3}$ Bipy ............................ 245

Fig. 7.20 - Espectro de excitação (Exc - linha vermelha) e espectro de absorção do composto $\left[\mathrm{Eu}(\mathrm{btfa})_{3}\right.$ bipy] depositado na forma de filme fino sobre quartzo. É evidente que ambos os espectros estão centrados em torno de $332 \mathrm{~nm}$, confirmando a posição em energia da excitação para este complexo na forma de filme. 246

Fig. 7.21 - Espectro de fotoluminescência $(F L)$ à temperatura ambiente do complexo [Eu(btfa)3bipy] sob a forma de filme fino depositado sobre quartzo. O espectro mostra as estreitas linhas de emissão do íon $\mathrm{Eu}^{3+}$,

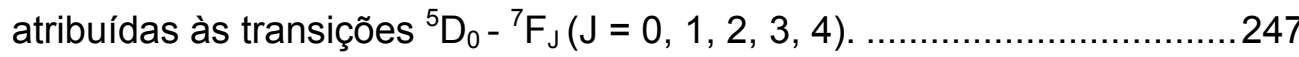

Fig. 7.22 - Eletroluminescência do dispositivo tricamada do complexo [Eu(btfa) ${ }_{3}$ bipy] em função da tensão aplicada. Como no caso da (FL) é possível identificar facilmente as transições características do íon $\mathrm{Eu}^{3+} \ldots 247$

Fig. 7.23 - Decaimento da intensidade da eletroluminescência do dispositivo sem a exposição da radiação U.V. Esta é a degradação natural desse dispositivo. A tensão é aplicada somente no momento de gravar o espectro. O gráfico inserido mostra que a intensidade inicial $\left({ }^{5} D_{0}-{ }^{7} F_{2}\right)$ cai à metade em aproximadamente 80 minutos. 248

Fig. 7.24 - Montagem esquemática para os experimentos de fotodegração dos filmes e Oleds baseados no complexo Eu(btfa)3bipy, onde, (1) Lâmpada de $\mathrm{Xe}$, (2) monocromador de excitação, (amostra) filme fino ou OLED, (3) monocromador de emissão e (4) detector (fotomultiplicadora).

Fig. 7.25 - Decaimento da intensidade da eletroluminescência do dispositivo entre doses de radiação U.V. Esta é a degradação natural desse dispositivo. A tensão é aplicada somente no momento de gravar o espectro. O gráfico inserido mostra que a intensidade inicial $\left({ }^{5} D_{0}-{ }^{7} F_{2}\right)$ cai à metade em aproximadamente 60 minutos para o filme (quadrados pretos) contra apenas 5 minutos para o dispositivo (triângulos azuis). 250 


\section{Lista de Tabelas}

Tabela 2.1 - Valores típicos de algumas propriedades do $\mathrm{Alq}_{3}{ }^{[36]}$ .55

Tabela 3.1 - Configuração eletrônica dos elementos terras-raras. Os colchetes representam a distribuição eletrônica do gás nobre correspondente..........76

Tabela 4.1 - Lista de compostos orgânicos usados durante a tese .....................91

Tabela 4.2 - Detalhes do processo de decapagem do ITO ...............................94

Tabela 4.3 - Fases e protótipos do processo de Fotolitografia ...........................98

Tabela 4.4 - Parâmetros utilizados durante o processo de deposição dos vários

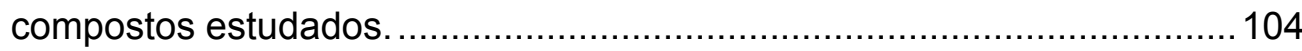

Tabela 4.5 - Detalhes dos dispositivos OLEDS construídos .........................107

Tabela 5.1 - Designação dos tipos de luminescência mais significativos.........111

Tabela 5.2 - Principais unidades de medidas Radiométricas e Fotométricas .. 123

Tabela 5.3 - Valores encontrados na literatura para os níveis HOMO e LUMO

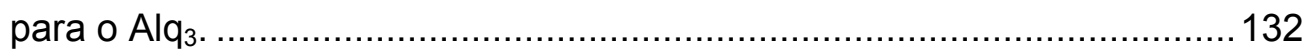

Tabela 7.1 - Porcentagem de fotodegradação para o complexo de Európio $\mathrm{Eu}(\mathrm{TTA})_{3}(\mathrm{TPPO})_{2}$ para excitações de $\mathrm{O}$ 1s, $\mathrm{C}$ 1s e valência. As porcentagens para as excitações do $S 2 p, S 2 s, P 2 p$ e $P$ 2s não estão relacionados na tabela por terem tido $100 \%$ de degradação. Todos os espectros foram aproximadamente normalizados à mesma linha de base. 


\section{Resumo}

Welber Gianini Quirino. Produção e caracterização de dispositivos orgânicos eletroluminescentes (OLEDs) baseados em complexos $\beta$ dicetonatos de Terras-raras. Rio de Janeiro, 2007. 260p. Tese de Doutorado - Departamento de Física, Pontifícia Universidade Católica do Rio de Janeiro.

Este trabalho apresenta os resultados de um estudo que envolve a fabricação e a caracterização de dispositivos orgânicos emissores de luz (OLEDs) baseados em complexos $\beta$-dicetonatos de terras-raras. $O$ estudo se coloca como continuação lógica da linha de pesquisa em dispositivos eletroluminescentes baseados em íons terras-raras, começada alguns anos atrás neste grupo de pesquisa. Para a produção dos dispositivos foram empregadas várias técnicas de deposição de filmes finos, tais como deposição térmica resistiva, pulverização catódica assistida por plasma (rf-magnetronsputtering) e spin-coating. A síntese dos compostos orgânicos, bem como alguns estudos adicionais puderam ser realizadas através de colaborações com diversos grupos de pesquisas nacionais, os quais dispõem de recursos e capacitação em áreas complementares. Os complexos orgânicos estudados foram divididos em três conjuntos, que chamamos de sistemas. No sistema 1 , estudou-se o complexo $\mathrm{Eu}(\mathrm{bmdm})_{3}(\mathrm{ttpo})_{2}$, onde o ligante orgânico bmdm é um conhecido agente absorvedor de radiação UV bastante usado em protetores solares. Os OLEDs baseados neste complexo apresentaram intensa foto- e eletroluminescência com alta pureza de cor dada apenas pelas finas transições características do íon $\mathrm{Eu}^{3+}$. No sistema 2, estudou-se o complexo chamado de binuclear. Este composto tem dois núcleos terras-raras coordenados numa mesma molécula. $\mathrm{O}$ primeiro binuclear estudado, o complexo $\mathrm{Eu}(\mathrm{btfa})_{3} \cdot$ phenterpy $\cdot \mathrm{Tb}(\mathrm{acac})_{3}$, não apresentou as transições características dos íons $\mathrm{Tb}^{3+}$ e $\mathrm{Eu}^{3+}$ como era esperado inicialmente. Por outro lado, apresentou uma eletroluminescência sintonizável em duas situações distintas, a primeira em função da tensão aplicada e a segunda através de mudanças na arquitetura das camadas constituintes. Por causa desse efeito, mostramos a possibilidade de se construir um dispositivo OLED emissor de luz branca. Ainda nesse sistema, foram estudados OLEDs com complexos modificados quimicamente, chamados de "binuclear 2" e trinuclear. O complexo binuclear 2 apresentou as linhas de emissão dos íons $\mathrm{Tb}^{3+}$ e $\mathrm{Eu}^{3+}$. Apesar de menos eficiente que o primeiro complexo binuclear, este estudo mostrou que através de manipulações 
moleculares (nanotecnologia) é possível sintetizar compostos capazes de emitir as linhas características de emissão dos íons terras-raras, ou seja, com um único complexo é possível obter duas emissões distintas. Por último, ainda como sistema 2, o complexo trinuclear, é uma mistura de compostos orgânicos contendo $\mathrm{Tm}$, Tb e Eu e não formam uma única molécula, como no caso dos compostos binucleares. Este estudo foi iniciado recentemente e ainda não foi completamente explorado. Os primeiros testes mostraram que é possível usar este complexo também para fabricar OLEDs com emissão de cor branca, variando-se as quantidades relativas de $\mathrm{Tm}, \mathrm{Tb}$ e Eu da mistura. Sabendo-se que os ligantes $\beta$-dicetonas são os responsáveis pela transferência de energia para os íons $\mathrm{TR}^{3+}$, através do efeito antena, o sistema 3 , despontou como grande novidade, mostrando a construção de dispositivos eletroluminescentes baseados em complexos tetrakis( $\beta$-dicetonatos) de TR, ou seja, compostos que possuem quatro ligantes $\beta$-dicetonas coordenandos a um único íon TR. Com esse sistema conseguimos pela primeira vez uma emissão eficiente e pura das principais transições do íon $\mathrm{Tb}$ à temperatura ambiente. $\mathrm{O}$ trabalho apontou, também, que tanto a irradiação com luz UV, quanto a exposição aos agentes atmosféricos (oxigênio, água, umidade, etc.) contribuem para uma rápida degradação dos complexos orgânicos com conseqüente decaimento do desempenho dos dispositivos fabricados. Para tanto, iniciamos um estudo para investigar as causas da degradação de alguns dos compostos orgânicos utilizados na fabricação de OLEDs. Os estudos de fotoabsorção e fotoemissão realizados no Laboratório Nacional de Luz Síncrotron foram fundamentais para uma maior compreensão destes efeitos. Os resultados deste estudo mostraram que as técnicas espectroscópicas empregadas neste trabalho podem ser utilizadas para se investigar a estrutura eletrônica, bem como a fotodegradação de compostos orgânicos usados na fabricação de OLEDs. Utilizando a espectroscopia de fluorescência, se estudou a viabilidade de se construir um dosímetro de radiação ultravioleta portátil e de uso pessoal usando um OLED cuja eletroluminescência é sensível à radiação UV.

\section{Palavras-chave}

Dispositivos eletroluminescentes orgânicos, OLEDs, eletroluminescência, complexos $\beta$-dicetonatos de Terras-Raras, filmes finos, nanotecnologia. 


\section{Abstract}

Welber Gianini Quirino. Production and characterization of organic electroluminescent devices (OLEDs) based on $\beta$-diceketone RareEarth complexes. Rio de Janeiro, 2007. 260p. PhD Thesis - Physics Departament, Pontifical Catolic University of Rio de Janeiro.

In this work we present the results of a study that involves the manufacture and the characterization of organic eletroluminescent devices (OLEDs) based on $\beta$-diketonates Rare-Earth complexes. The investigation reported is a continuation of the research in electroluminescent devices based on rare-earth ions, started some years ago in our Group. For the production of the devices were applied several thin films deposition techniques: thermal resistive, rf-magnetronsputtering and spin-coating. The synthesis of organic compounds, as well some additional studies, were carried on through the collaboration with different brazilian research groups, which have resources and qualification in complementary areas. The organic compounds studied in this thesis have been divided in three groups, named systems. In system 1, the studied complex was $\mathrm{Eu}(\mathrm{bmdm})_{3}(\mathrm{ttpo})_{2}$, where the organic bmdm ligand is a known UV sensitive material, frequently used in sunblockers. The OLEDs based on this complex presented intense photo- and electroluminescence with high pure color emission due to the almost atomic transitions characteristic of the $\mathrm{Eu}^{3+}$ ion. In system 2, a binuclear complex, represented by the molecular formula $\mathrm{Eu}(\mathrm{btfa})_{3} \cdot$ phenterpy $\cdot \mathrm{Tb}(\mathrm{acac})_{3}$ was studied. This complex has two rare-earth nuclei coordinated in the same molecule. The OLEDs based on this complex did not present the $\mathrm{Tb}^{3+}$ and $\mathrm{Eu}^{3+}$ characteristic transitions as expected. On the other hand, the complex gave us the possibility to develop an OLED with white color emission. Probably the major novelty of this thesis is represented by system 3 . Indeed, knowing that the $\beta$-diketone ligands are the main responsible for the $\mathrm{RE}^{3+}$ ions energy transference through the antenna effect, in system 3 , we investigated the possibility to fabricate electroluminescent devices based on RE $\beta$-diketonate tetrakis complexes, which have four coordinated $\beta$-diketones ligand to an RE ion in order to enchance the energy transfer and the emission efficiency. With this system we obtained, for the first time, an efficient and pure Tb emission at room temperature.

\section{Keywords}

Organic Eletroluminescent devices, OLEDs, eletroluminescence, RareEarths $\beta$-diketonates complexes, thin films, nanotecnology. 


\section{Lista de Símbolos e Abreviações}

EL - Eletroluminescência; FL - Fotoluminescência

LEDs - Light Emission Diodes

HTL - Transportador de buracos

ETL - Transportador de elétrons

OLEDs - Dispositivos emissores de luz, do inglês Organic Light Emission

Diodes

LOEM - Laboratório de Optoeletrônica Molecular

SMOLEDs - Oleds baseados em pequenas moléculas, do inglês Small

Molecule Organic Light Emission Diodes

POLEDs - Oleds baseados em polímeros, do inglês Polymer Organic

Light Emission Diodes

HOMO - Orbital molecular mais alto ocupado, do inglês Highest Occupied

Molecular Orbital

LUMO - Orbital molecular mais baixo desocupado, do inlgês Lowest

Unoccupied Molecular Orbital

TCO - Óxidos transparentes condutores

TCL - Transporte de cargas limitado por armadilhas

SCL - Transporte de carga limitado por carga espacial

TFL - Limite de transporte de armadilha cheia

$\varepsilon$ - Campo elétrico aplicado

$\Delta$ - Diferença de energia entre a função trabalho do metal e o nível

molecular

$\Phi$ - Energia do nível molecular

J - Densidade de corrente

$h$ - Constante de Planck

$k$ - Constante de Boltzmann

$\varphi$ - Barreira de potencial para injeção de portadores

$E_{T}$ - Nível de energia das armadilhas em relação ao LUMO

$\mu$ - Mobilidade dos portadores 